\title{
Viscous Hydrodynamic Predictions for Nuclear Collisions at the LHC
}

\author{
Matthew Luzum ${ }^{1, *}$ and Paul Romatschke, \\ ${ }^{1}$ Department of Physics, University of Washington, Seattle, WA 98195-1560 \\ ${ }^{2}$ Institute for Nuclear Theory, University of Washington, Seattle, WA 98195-1560
}

(Dated: October 15, 2018)

\begin{abstract}
Hydrodynamic simulations are used to make predictions for the integrated elliptic flow coefficient $v_{2}$ in $\sqrt{s}=5.5 \mathrm{TeV}$ lead-lead and $\sqrt{s}=14 \mathrm{TeV}$ proton-proton collisions at the LHC. We predict a $10 \%$ increase in $v_{2}$ from RHIC to $\mathrm{Pb}+\mathrm{Pb}$ at LHC, and $v_{2} \sim 0$ in $\mathrm{p}+\mathrm{p}$ collisions unless $\eta / s<0.08$.
\end{abstract}

\section{INTRODUCTION}

Much work has been done recently using viscous hydrodynamics to study the properties of gold-gold collisions at the Relativistic Heavy Ion Collider (RHIC) [1 [4]. A measurement of particular interest is the elliptic flow coefficient $v_{2}$, the second moment in the azimuthal angle of the distribution of emitted particles (cf. [5]), which allows to extract information about material constants (such as viscosity) of the high density nuclear matter created at RHIC. Using the knowledge gained at RHIC, it should be possible to predict experimental results at the Large Hadron Collider (LHC), which will collide lead ions at a maximum center of mass energy of $\sqrt{s}=5.5 \mathrm{TeV}$ per nucleon pair compared to $\sqrt{s}=200 \mathrm{GeV}$ gold ions at RHIC. If experimental data on, e.g., $v_{2}$ from LHC is close to the hydrodynamic model prediction, this would confirm that real progress has been made in understanding nuclear matter at extreme energy densities; if far away, it may indicate that the successful hydrodynamic description of experimental data from RHIC was a coincidence.

Regardless of the outcome, the advent of the RHIC experiments clearly has lead to major progress in the theory and application of hydrodynamics to heavy-ion collisions. A few years ago the form of the hydrodynamic equations in the presence of shear viscosity $\eta$ was still unresolved, with different groups keeping some terms while neglecting others [6 9]. For the case of approximately conformal theories, where the viscosity coefficient for bulkbut not shear-becomes negligible, all possible terms to second order in gradients were derived in Ref. 10], and their relative importance investigated in Ref. [1]. Three of the groups performing viscous hydrodynamic simulations now agree on these terms [1, 3, 4], while another group 2] uses a different formalism that gives matching results. While this development still leaves out the consistent treatment of bulk viscosity, the quantitative suppression of elliptic flow by shear viscosity is therefore essentially understood. From comparison of viscous hydrodynamic simulations to experimental data [11, 12], one can infer an upper limit of the ratio of shear viscosity over entropy density, $\eta / s<0.5$, for the matter produced in $\mathrm{Au}+\mathrm{Au}$ collisions at $\sqrt{s}=200 \mathrm{GeV}$ [1], comprising extractions by other methods [13 15]. A sizeable uncertainty for this limit comes from the fact that the initial conditions for the hydrodynamic evolution are poorly known, with the two main models, the Glauber and Color-Glass-Condensate (CGC) models, giving different results for the elliptic flow coefficient [1]. This difference can be understood to originate from the different initial spatial eccentricity $e_{x}$ in the Glauber/CGC models. The eccentricity is defined as

$$
e_{x} \equiv \frac{\left\langle y^{2}-x^{2}\right\rangle}{\left\langle y^{2}+x^{2}\right\rangle}
$$

where the symbols \langle\rangle denote averaging over the initial energy density in the transverse plane, $\epsilon(x, y)$.

Indeed, it had been suggested [16] that the elliptic flow coefficient $v_{2}$ at the end of the hydrodynamic evolution would be strictly proportional to the initial spatial eccentricity, $v_{2} / e_{x} \propto$ const., if the fluid was evolving without any viscous stresses for an infinitely long time. This is to be contrasted with experimental data indicating a proportionality factor of total multiplicity over overlap area

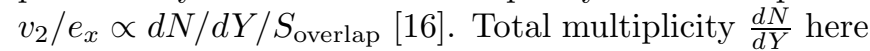
refers to the total number of observed particles $N$ per unit rapidity $Y$, while the overlap area is calculated as

$$
S_{\text {overlap }}=\pi \sqrt{\left\langle x^{2}\right\rangle\left\langle y^{2}\right\rangle} .
$$

Ideal fluid dynamics does not adequately describe the latest stage of a heavy-ion collision (the hadron gas), because of the large viscosity coefficient in this stage [17]. Therefore, the hydrodynamic stage lasts only for a finite time (e.g. until all fluid cells have cooled below the decoupling temperature), resulting in a dependence of $v_{2} / e_{x}$ on $d N / d Y$. Also, viscous effects affect the proportionality between $v_{2}$ and $e_{x}$, leading to a behavior that is qualitatively similar to that observed in the data [4].

One of the objectives of this work is to extend the energy range for fluid dynamic results of $v_{2} / e_{x}$ from $\mathrm{Au}+\mathrm{Au}$ collisions at top $\mathrm{RHIC}$ to $\mathrm{Pb}+\mathrm{Pb}$ collisions at top $\mathrm{LHC}$ energies, as well as to study the dependence on shear viscosity. If in the future either $e_{x}$ or the mean $\eta / s$ becomes known, these results can thus be used to constrain the respective other quantity from experimental data. On the other hand, the values of shear viscosity for which the Glauber/CGC models match to experimental data at top RHIC energies have been extracted in Ref. [1, 18] for $\mathrm{Au}+\mathrm{Au}$ collisions. Since $\eta / s$ averaged over the system 
evolution is not expected to be dramatically different for $\mathrm{Pb}+\mathrm{Pb}$ collisions at the LHC, another objective of this work is to obtain a prediction for the elliptic flow coefficient for the LHC based on the best-fit values to RHIC.

Finally, the feasibility of detecting elliptic flow in $\mathrm{p}+\mathrm{p}$ collisions at $\sqrt{s}=14 \mathrm{TeV}$ at the LHC is being discussed [19]. As a reference for other approaches and experiment, it interesting to study the possible size and viscosity dependence of $v_{2}$ under the hypothetical assumption that the bulk evolution following $\mathrm{p}+\mathrm{p}$ collisions could be captured by fluid dynamics.

\section{SETUP}

To make predictions for nuclear collisions at LHC energies, we use our hydrodynamic model that successfully described experimental data at RHIC [1, 18] and make modifications to the input parameters appropriate for the higher collision energies at the LHC.

As a reminder, the hydrodynamic model [1] is based on the conservation of the energy momentum tensor [10]

$$
\begin{aligned}
& T^{\mu \nu}=\epsilon u^{\mu} u^{\nu}-p \Delta^{\mu \nu}+\Pi^{\mu \nu}, \\
& \Pi^{\mu \nu}=\eta \nabla^{\langle\mu} u^{\nu\rangle}-\tau_{\Pi}\left[\Delta_{\alpha}^{\mu} \Delta_{\beta}^{\nu} D \Pi^{\alpha \beta}+\frac{4}{3} \Pi^{\mu \nu}\left(\nabla_{\alpha} u^{\alpha}\right)\right] \\
& -\frac{\lambda_{1}}{2 \eta^{2}} \Pi^{<\mu}{ }_{\lambda} \Pi^{\nu>\lambda}+\frac{\lambda_{2}}{2 \eta} \Pi^{<\mu}{ }_{\lambda} \omega^{\nu>\lambda}-\frac{\lambda_{3}}{2} \omega^{<\mu}{ }_{\lambda} \omega^{\nu>\lambda},
\end{aligned}
$$

where $\epsilon, p$ and $u^{\mu}$ are the energy density, pressure, and fluid 4-velocity, respectively. $D \equiv u^{\mu} D_{\mu}$ and $\nabla_{\alpha} \equiv \Delta_{\alpha}^{\mu} D_{\mu}$ are time-like and space-like projections of the covariant derivative $D_{\mu}$, where $\Delta^{\mu \nu}=$ $g^{\mu \nu}-u^{\mu} u^{\nu}$ and we remind the compact notations $A_{\langle\mu} B_{\nu\rangle} \equiv\left(\Delta_{\mu}^{\alpha} \Delta_{\nu}^{\beta}+\Delta_{\nu}^{\alpha} \Delta_{\mu}^{\beta}-\frac{2}{3} \Delta^{\alpha \beta} \Delta_{\mu \nu}\right) A_{\alpha} B_{\beta}$ and $\omega_{\mu \nu} \equiv \frac{1}{2}\left(\nabla_{\nu} u_{\mu}-\nabla_{\mu} u_{\nu}\right)$. For relativistic nuclear collisions it is convenient to follow Bjorken [20] and use Milne coordinates proper time $\tau=\sqrt{t^{2}-z^{2}}$ and spacetime rapidity $\xi=\operatorname{atanh} \frac{z}{t}$, in which the metric becomes $g_{\mu \nu}=\operatorname{diag}\left(1,-1,-1,-\tau^{2}\right)$, and assume that close to $\xi=0$, the hydrodynamic degrees of freedom are approximately boost-invariant $(\xi \simeq Y)$.

The hydrodynamic equations $D_{\mu} T^{\mu \nu}=0$ then constitute an initial value problem in proper time and transverse space, and are solved numerically (see Ref. [1]). The input parameters for hydrodynamic evolution are the equation of state $p=p(\epsilon)$ and the first (second) order hydrodynamic transport coefficients $\eta\left(\tau_{\Pi}, \lambda_{1}, \lambda_{2}, \lambda_{3}\right)$. The values for $\lambda_{1,2,3}$ have been found to hardly affect the boost-invariant hydrodynamic evolution for $\mathrm{Au}+\mathrm{Au}$ collisions at RHIC [1], so here they are generally set to zero.

The mechanisms leading to thermalization (the onset of hydrodynamic behavior) are not well understood in nuclear collisions. Therefore, it is not known how the thermalization time $\tau_{0}$ at which hydrodynamic evolution is started depends on the collision energy. Barring further

\begin{tabular}{|cccccc|}
\hline \hline Beam & Initial cond. $\frac{d N_{\text {ch }}}{d Y}$ & $T_{i}[\mathrm{GeV}]$ & $\sqrt{s}[\mathrm{GeV}]$ & $\tau_{0}[\mathrm{fm} / \mathrm{c}]$ \\
\hline Gold & Glauber & 800 & 0.34 & 200 & 1 \\
Gold & CGC & 800 & 0.31 & 200 & 1 \\
Lead & Glauber & 1800 & 0.42 & 5500 & 1 \\
Lead & CGC & 1800 & 0.39 & 5500 & 1 \\
Protons & Glauber & 6 & 0.400 & 14000 & 0.5 \\
Protons & Glauber & 6 & 0.305 & 14000 & 1 \\
Protons & Glauber & 6 & 0.270 & 14000 & 2 \\
\hline \hline
\end{tabular}

TABLE I: Central collision parameters used for the viscous hydrodynamics simulations $\left(T_{f}=0.14 \mathrm{GeV}\right.$ for all).

insight, we start hydrodynamic evolution for the LHC at the same time as for the RHIC simulations $\left(\tau_{0}=1 \mathrm{fm} / \mathrm{c}\right)$. At this time, the initial conditions for the transverse energy density $\epsilon(x, y)$ are given by the Glauber or CGC model, respectively, the fluid velocities are assumed to vanish, and the shear tensor $\Pi^{\mu \nu}$ is set to zero (other values for $\Pi^{\mu \nu}$ do not seem to affect the final results [1, 21]). For brevity, we refer to Ref. [1] for the details of the Glauber and CGC models, but for the expert reader note that we use the Woods-Saxon parameters of radius $R_{0}=6.4(6.6) \mathrm{fm}$ and skin depth $\chi=0.54(0.55) \mathrm{fm}$ for gold (lead), and assume a nucleon-nucleon cross section of $\sigma=40(60) \mathrm{mb}$ for $\sqrt{s}=200(5500) \mathrm{GeV}$ collisions.

The overall normalization of the initial energy density (parametrized by the initial temperature at the center $T_{i}$ ) was adjusted to match the experimentally observed multiplicity at RHIC; by analogy, for LHC the normalization is adjusted to match the predicted multiplicity [22 25]. Since we lack detailed knowledge about its temperature dependence, the ratio of shear viscosity to entropy density $\eta / s$ is set to be constant during the hydrodynamic evolution (equal to the average over the spacetime evolution of the system). The relaxation time coefficient $\tau_{\Pi}$ is expected [10, 26] to lie in the range $\frac{\tau_{\Pi}}{\eta}(\epsilon+p) \simeq 2.6-6$. The equation of state $(\mathrm{EoS})$ can in principle be provided by lattice QCD. While at present there are points of disagreement between lattice groups about, e.g., the precise location of the QCD phase transition, there is consensus that it is an analytic crossover [27, 28]. Therefore, we use a lattice-inspired EoS [29] that is consistent with both the current consensus and perturbative QCD; also, since it resembles [27], we expect that using a different lattice EoS will have a minor effect on our results.

Once a given fluid cell has cooled down to the decoupling temperature $T_{f}$, its energy and momentum are converted into particle degrees of freedom using the CooperFrye freeze-out prescription [30]. A value of $T_{f}=0.14$ $\mathrm{GeV}$ was determined by matching to RHIC data and will also be used for LHC energies, assuming that it is mostly determined by local conditions, and less so by initial energy density, system size or collision energy. The distribution of the particle degrees of freedom may be further 


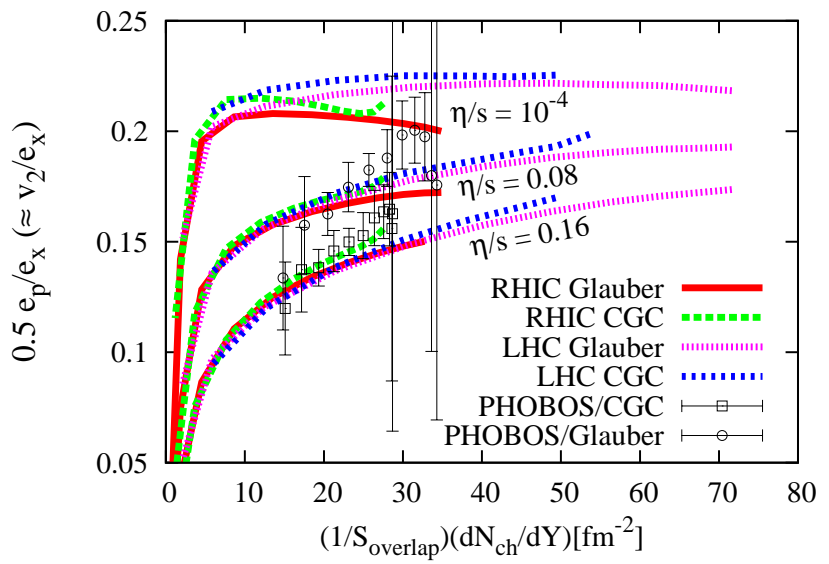

FIG. 1: (Color online) Anisotropy (3) divided by (1), as a function of initial entropy (44) divided by (2). Shown are results from hydrodynamic simulations for $\sqrt{s}=200 \mathrm{GeV}$ $\mathrm{Au}+\mathrm{Au}$ (RHIC) and $\sqrt{s}=5.5 \mathrm{TeV} \mathrm{Pb}+\mathrm{Pb}$ collisions (LHC). For comparison, experimental data for $v_{2}$ from RHIC [31], divided by $e_{x}$ from two models [13], is shown as a function of measured $\frac{d N_{\mathrm{ch}}}{d Y}$ [32] divided by (2). See text for details.

evolved using a hadronic cascade code (as in Ref. 33]), or in a more simple approach the unstable particle resonances are allowed to decay, without further evolving the stable particle distributions. In both cases, the total multiplicity and particle correlations (such as the elliptic flow coefficient) are then calculated from the stable particle distribution (cf. [1]). Surprisingly, it was found in Ref. [1, 5] that the momentum integrated elliptic flow coefficient for charged hadrons - to good approximation - is equal to half the momentum anisotropy,

$$
v_{2} \simeq \frac{1}{2} e_{p}=\frac{1}{2} \frac{\int d x d y T^{x x}-T^{y y}}{\int d x d y T^{x x}+T^{y y}} .
$$

Since the momentum anisotropy is a property of the fluid, it is independent on the details of the freeze-out procedure and only mildly dependent on the choices of $\tau_{0}, T_{f}$. Unlike at RHIC where pairs of $\tau_{0}$ and $T_{f}$ could be finetuned to fit the particle spectra at central collisions, no such extra information is available for the LHC. Hence Eq. (3) may provide the most reliable way of determining the elliptic flow of charged hadrons, and will be used in the following. Similarly, one can use the total entropy per unit spacetime rapidity $\frac{d S}{d \xi}$ in the fluid as a proxy for the total (charged hadron) multiplicity per unit rapidity $\frac{d N}{d Y}\left(\frac{d N_{\mathrm{ch}}}{d Y}\right)$ with a proportionality factor 34,35$]$

$$
\frac{d S}{d \xi} \sim \frac{d S}{d Y} \simeq 4.87 \frac{d N}{d Y} \simeq 7.85 \frac{d N_{\mathrm{ch}}}{d Y} .
$$

Note that for a gas of massive hadrons in thermal equilibrium at $T_{f}=0.14 \mathrm{GeV}$ the ratio of entropy to particle density is $\sim 6.41$, but the decay of unstable resonances produces additional entropy, resulting in Eq. (4).

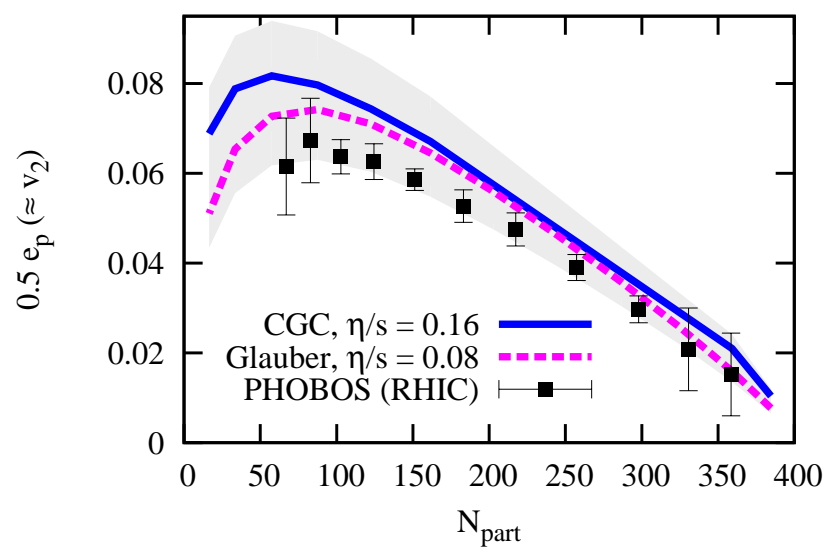

FIG. 2: (Color online) Anisotropy (3) prediction for $\sqrt{s}=5.5$ $\mathrm{TeV} \mathrm{Pb}+\mathrm{Pb}$ collisions (LHC), as a function of centrality. Prediction is based on values of $\eta / s$ for the Glauber/CGC model that matched $\sqrt{s}=200 \mathrm{GeV} \mathrm{Au}+\mathrm{Au}$ collision data from PHOBOS at RHIC (31], shown for comparison). The shaded band corresponds to the estimated uncertainty in our prediction from additional systematic effects: using $e_{p} / 2$ rather than $v_{2}(5 \%)$ [1]; using a lattice EoS from [29] rather than 27] (5\%); not including hadronic cascade afterburner (5\%) [38]

Since results from RHIC suggest there is only approximately $10 \%$ viscous entropy production during the hydrodynamic phase [4, 36], the entropy $\frac{d S}{d Y}$ at $\tau=\tau_{0}$ can be used to estimate the final particle multiplicity. In the case of the LHC, the world average for the predicted charged hadron multiplicity for central $\mathrm{Pb}+\mathrm{Pb}$ collisions at $\sqrt{s}=5.5 \mathrm{TeV}$ [22], $\frac{d N_{\text {ch }}}{d Y} \simeq 1800$, can be used to estimate the total entropy at $\tau=\tau_{0}$, and hence the overall normalization $T_{i}$ of the initial energy density (see Tab. II).

Using Eqs. (314) for the multiplicity and elliptic flow allows to make predictions for the LHC without having to model the hadronic freeze-out, which should make the results more robust. However, as a consequence one does not get information about the momentum dependence of the elliptic flow coefficient, prohibiting detailed comparison with predictions by other groups [23, 37].

\section{RESULTS}

With the initial energy density distribution fixed at $\tau_{0}$, the hydrodynamic model then gives predictions for the ratio of $v_{2} / e_{x}$ at the LHC. In Fig. 11, the results are shown for three different values of shear viscosity, for two different initial conditions and two different beams/collision energies $(\mathrm{Au}+\mathrm{Au}$ at $\sqrt{s}=200 \mathrm{GeV}, \mathrm{Pb}+\mathrm{Pb}$ at $\sqrt{s}=5.5$ $\mathrm{TeV})$. The resulting values for $v_{2} / e_{x}$ seem to be quasiuniversal functions of the total multiplicity scaled by the overlap area $S_{\text {overlap }}$, only depending on the value of $\eta / s$ (and, to a lesser extent, the collision energy). The devi- 
ations of the RHIC simulations from the universal curve can be argued to arise from a combination of the finite lifetime of the hydrodynamic phase at $\sqrt{s}=200$ $\mathrm{GeV}$ and the presence of the QCD phase transition, and is strongest for ideal hydrodynamics, in agreement with earlier findings [4].

Also shown in Fig. 1 is experimental data for the elliptic flow coefficient for $\mathrm{Au}+\mathrm{Au}$ collisions at $\mathrm{RHIC}$, normalized by $e_{x}$ from a Monte-Carlo calculation (including fluctuations) in Glauber and CGC models (see Ref. [13] for details). Since $e_{x}$ is not directly measurable, the differently normalized data gives an estimate of the overall size of $v_{2} / e_{x}$ at RHIC. Directly matching experimental data on $v_{2}$ using a hydrodynamic model with an initial $e_{x}$ specified by the Glauber or CGC model, a reasonable fit was achieved for a mean value of $\eta / s \simeq 0.08$ and $\eta / s \simeq 0.16$, respectively [1, 18]. Under the assumption that the average $\eta / s$ is similar for collisions at RHIC and the LHC (along with the assumptions discussed above), one can make a prediction for the integrated elliptic flow coefficient for charged hadrons as a function of impact parameter (or more customarily the number of participants $N_{\text {part }}$, cf. [1]). The result is shown in Fig. 22. As can be seen, we expect integrated $v_{2}$ at the LHC to be about ten percent larger than at RHIC, which is less than the prediction by ideal hydrodynamics [39], and in agreement with the extrapolations by Drescher et al. 23].

Finally, using the charge density parametrization of the proton $\rho(b)$ in Ref. [40] as an equivalent of the nuclear thickness function in the Glauber model (cf. [1]) one obtains an estimate for the shape of the transverse energy density following a relativistic $\mathrm{p}+\mathrm{p}$ collision. Using the predicted multiplicity at mid-rapidity $\frac{d N}{d Y} \sim 6$ [24, 25] for $\sqrt{s}=14 \mathrm{TeV} \mathrm{p}+\mathrm{p}$ collisions at the LHC, one can again use Eq. (41) to infer the overall normalization of the energy density (or $T_{i}$ ) at $\tau=\tau_{0}$ (see Tab. I). As a "Gedankenexperiment" one can then ask how much elliptic flow would be generated in LHC p+p collisions if the subsequent evolution was well approximated by boost-invariant viscous hydrodynamics. One finds that for ideal hydrodynamics $\frac{e_{p}}{2} \sim v_{2} \sim 0.035$ for integrated $\left|v_{2}\right|$ in minimum bias collisions (cf. (28) in [1]), while for $\eta / s \geq 0.08, v_{2}$ typically changes by almost 100 percent when varying the relaxation time $\frac{\tau_{\Pi}}{\eta}(\epsilon+p)$ between 2.6 and 6 and varying $\tau_{0}$ by a factor of two. This indicates that for $\eta / s \geq 0.08$, the hydrodynamic gradient expansion does not converge and as a consequence it is unlikely that elliptic flow develops in $\mathrm{p}+\mathrm{p}$ collisions at top LHC energies. If experiments find a non-vanishing value for integrated $\left|v_{2}\right|>0.02$ in minimum bias $\mathrm{p}+\mathrm{p}$ collisions, this would be an indication for an extremely small viscosity $\eta / s<0.08$ in deconfined nuclear matter.

To conclude, viscous hydrodynamics can be used to make predictions for the ratio of $v_{2} / e_{x}$ as a function of multiplicity and $\eta / s$. Assuming a multiplicity of $\frac{d N_{\mathrm{ch}}}{d Y} \simeq 1800$ for the matter created in $\mathrm{Pb}+\mathrm{Pb}$ collisions at LHC, as well as $\eta / s$ similar to RHIC, we predict the integrated elliptic flow for charged hadrons to be $10 \%$ larger at the LHC than at RHIC. We expect $v_{2}$ measurements in $\mathrm{p}+\mathrm{p}$ collisions to be consistent with zero, unless the shear viscosity is extremely small $(\eta / s<0.08)$.

We would like to thank J.-Y. Ollitrault for providing tabulated results from 13], and J. Albacete, K. Eskola, G.A. Miller, L. Ramello, J. Schukraft, R. Snellings and P. Steinberg for discussions. The work of ML and PR was supported by the US Department of Energy, grant numbers DE-FG02-97ER41014 and DE-FG02-00ER41132, respectively.

* Electronic address: mluzum@phys.washington.edu

† Electronic address: paulrom@phys.washington.edu

[1] M. Luzum and P. Romatschke, Phys. Rev. C78, 034915 (2008).

[2] K. Dusling and D. Teaney, Phys. Rev. C77, 034905 (2008).

[3] P. Huovinen and D. Molnar, Phys. Rev. C79, 014906 (2009).

[4] H. Song and U. W. Heinz, Phys. Rev. C78, 024902 (2008).

[5] P. F. Kolb, J. Sollfrank, and U. W. Heinz, Phys. Lett. B459, 667 (1999).

[6] A. Muronga, Phys. Rev. C69, 034903 (2004).

[7] U. W. Heinz, H. Song, and A. K. Chaudhuri, Phys. Rev. C73, 034904 (2006).

[8] R. Baier, P. Romatschke, and U. A. Wiedemann, Phys. Rev. C73, 064903 (2006).

[9] T. Koide, G. S. Denicol, P. Mota, and T. Kodama, Phys. Rev. C75, 034909 (2007).

[10] R. Baier, P. Romatschke, D. T. Son, A. O. Starinets, and M. A. Stephanov, JHEP 04, 100 (2008).

[11] B. Alver et al. (PHOBOS) (2007).

[12] B. I. Abelev et al. (STAR), Phys. Rev. C77, 054901 (2008).

[13] H.-J. Drescher, A. Dumitru, C. Gombeaud, and J.-Y. Ollitrault, Phys. Rev. C76, 024905 (2007).

[14] S. Gavin and M. Abdel-Aziz, Phys. Rev. Lett. 97, 162302 (2006).

[15] A. Adare et al. (PHENIX), Phys. Rev. Lett. 98, 172301 (2007).

[16] C. Alt et al. (NA49), Phys. Rev. C68, 034903 (2003).

[17] M. Prakash, M. Prakash, R. Venugopalan, and G. Welke, Phys. Rept. 227, 321 (1993).

[18] M. Luzum and P. Romatschke, Phys. Rev. C79, 039903(E) (2009).

[19] L. Ramello, private communication.

[20] J. D. Bjorken, Phys. Rev. D27, 140 (1983).

[21] H. Song and U. W. Heinz, Phys. Rev. C77, 064901 (2008).

[22] N. Armesto, J. Phys. G35, 104042 (2008).

[23] N. Armesto (ed) et al., J. Phys. G35, 054001 (2008).

[24] W. Busza, J. Phys. G35, 044040 (2008).

[25] D. Kharzeev, E. Levin, and M. Nardi, Nucl. Phys. A747, 609 (2005).

[26] M. A. York and G. D. Moore (2008), 0811.0729.

[27] A. Bazavov et al. (2009), 0903.4379. 
[28] Y. Aoki et al. (2009), 0903.4155.

[29] M. Laine and Y. Schroder, Phys. Rev. D73, 085009 (2006).

[30] F. Cooper and G. Frye, Phys. Rev. D10, 186 (1974).

[31] B. Alver et al. (PHOBOS), Phys. Rev. Lett. 98, 242302 (2007).

[32] S. S. Adler et al. (PHENIX), Phys. Rev. C69, 034909 (2004).

[33] S. A. Bass and A. Dumitru, Phys. Rev. C61, 064909 (2000).

[34] M. Gyulassy and T. Matsui, Phys. Rev. D29, 419 (1984).
[35] P. F. Kolb, U. W. Heinz, P. Huovinen, K. J. Eskola, and K. Tuominen, Nucl. Phys. A696, 197 (2001).

[36] P. Romatschke, Eur. Phys. J. C52, 203 (2007).

[37] A. K. Chaudhuri, Phys. Lett. B672, 126 (2009).

[38] D. Teaney, J. Lauret, and E. V. Shuryak (2001), nuclth/0110037.

[39] H. Niemi, K. J. Eskola, and P. V. Ruuskanen, Phys. Rev. C79, 024903 (2009).

[40] G. A. Miller, Phys. Rev. Lett. 99, 112001 (2007). 